- The osteoblast is the pivotal cell in bone remodelling and the link between the osteoblast and osteoclast recruitment and activation is now established

- Excessive orthodontic forces cause inefficient tooth movement and adverse tissue reactions

- The mechanisms which prevent root resorption are not fully understood but it remains a consequence of any orthodontic treatment. The extent and degree of root resorption cannot be predicted but some indicators are available

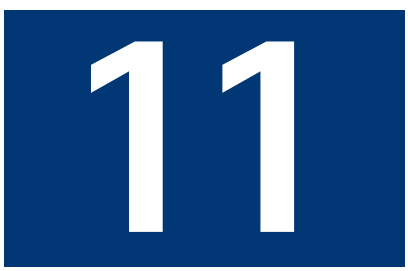

\title{
Orthodontics. Part 11: Orthodontic tooth movement
}

\author{
D. Roberts-Harry ${ }^{1}$ and J. Sandy ${ }^{2}$
}

\author{
NOW AVAILABLE \\ AS A BDJ BOOK
}

Orthodontic tooth movement is dependent on efficient remodelling of bone. The cell-cell interactions are now more fully understood and the links between osteoblasts and osteoclasts appear to be governed by the production and responses of osteoprotegerin ligand. The theories of orthodontic tooth movement remain speculative but the histological documentation is unequivocal. A periodontal ligament placed under pressure will result in bone resorption whereas a periodontal ligament under tension results in bone formation. This phenomenon may be applicable to the generation of new bone in relation to limb lengthening and cranialsuture distraction. It must be remembered that orthodontic tooth movement will result in root resorption at the microscopic level in every case. Usually this repairs but some root characteristics apparent on radiographs before treatment begins may be indicative of likely root resorption. Some orthodontic procedures (such as fixed appliances) are also known to cause root resorption.

\section{ORTHODONTICS \\ 1. Who needs orthodontics? \\ 2. Patient assessment and examination I \\ 3. Patient assessment and examination II \\ 4. Treatment planning \\ 5. Appliance choices \\ 6. Risks in orthodontic treatment \\ 7. Fact and fantasy in orthodontics \\ 8. Extractions in orthodontics \\ 9. Anchorage control and distal movement \\ 10. Impacted teeth \\ 11. Orthodontic tooth movement \\ 12. Combined orthodontic treatment}

${ }^{1 *}$ Consultant Orthodontist, Orthodontic Department, Leeds Dental Institute, Clarendon Way, Leeds LS2 9LU;

${ }^{2}$ Professor of Orthodontics, Division of

Child Dental Health, University of Bristol Dental School, Lower Maudlin Street, Bristol BS1 2LY;

${ }^{*}$ Correspondence to: D. Roberts-Harry E-mail: robertsharry@btinternet.com

\section{Refereed Paper}

doi:10.1038/sj.bdj.4811129

( ) British Dental Journal 2004; 196 :

391-394
The histological changes which occur when forces are applied to teeth are well documented (Figs 1 and 2). Teeth appear to lie in a position of balance between the tongue and lips or cheeks. This zone is not completely neutral since tongue forces are usually slightly greater than the lips or cheeks. The periodontal ligament is thought to have an intrinsic force which has to be overcome before teeth move. A notable feature of periodontal disease, where this intrinsic force is lost, is splaying, drifting and spacing of teeth. Similarly, if there is excessive tongue activity or destruction of the lips or cheeks (as in cancrum oris) then the teeth will drift.

Very low forces are capable of moving teeth. Classically, ideal forces in orthodontic tooth movement are those which just overcome capillary blood pressure. In this situation bone resorption is seen on the pressure side and bone deposition on the tension side. Teeth rarely move in this ideal way. Usually force is not applied evenly and teeth move by a series of tipping and uprighting movements. In some areas excessive pressure results in hyalanization where the cellular component of the periodontal ligament disappears. The hyalanized zone assumes a ground glass appearance but this returns to normal once the pressure is reduced and the periodontal ligament repopulated with normal cells. In this situation a different type of resorption is seen whereby osteoclasts appear to 'undermine' bone rather than resorbing at the 'frontal' edge (Fig. 3).

Mechanically induced remodelling is not fully understood. The role of the periodontal ligament has been questioned since tooth movement can still occur even where the peri- odontal ligament is not functioning normally. The ligament itself undergoes remodelling and the role of matrix metalloproteinases (MMPs) together with their natural inhibitors, tissue inhibitors of metalloproteinases (TIMPs) are clearly of importance. ${ }^{1}$

Osteocytes (osteoblasts incorporated into mineralized bone matrix) are situated in a rigid matrix and are thus ideally positioned to detect changes in mechanical stresses. They could signal to surface lining osteoblasts and thus bone formation and indeed bone resorption may result. There is now good understanding of key mechanisms in bone resorption and formation. Bone is formed by osteoblasts which also have a role in bone resorption. It is the osteoblast which has receptors for many of the hormones and growth factors which stimulate bone turnover.

By contrast, the osteoclast which resorbs mineralised tissue, responds to very few direct hormone actions. Most of the classic agents which have direct effects on osteoclasts have inhibitory actions. For example, Calcitonin and prostaglandin E2 will inhibit osteoclasts from resorbing calcified matrices.

The recruitment and activation of osteoclasts to sites of resorption comes from the osteoblast when the latter cell is stimulated by various hormones. The signal link from osteoblasts has recently been identified as osteoprotegerin (OPG) and the ligand (OPGL). They both potently inhibit and stimulate respectively, osteoclast differentiation. Furthermore, OPGL appears to have direct effects on stimulating mature osteoclasts into activity. If OPGL is injected into mice there is an 


\section{PRACTICE}

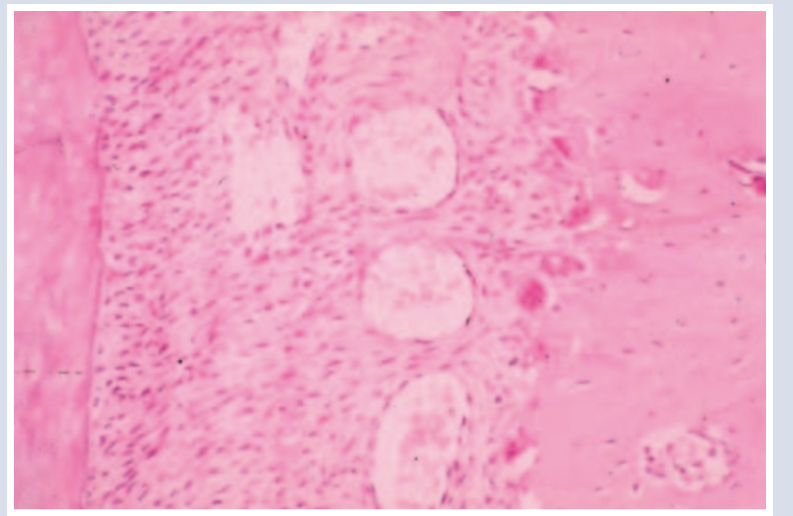

Fig. 1 Pressure side of a tooth being moved. The very vascular periodontal ligament has cementum on one side and bone on the other where frontal resorption is occurring. Osteoclasts can be seen in their lacunae resorbing bone on it's 'frontal edge'

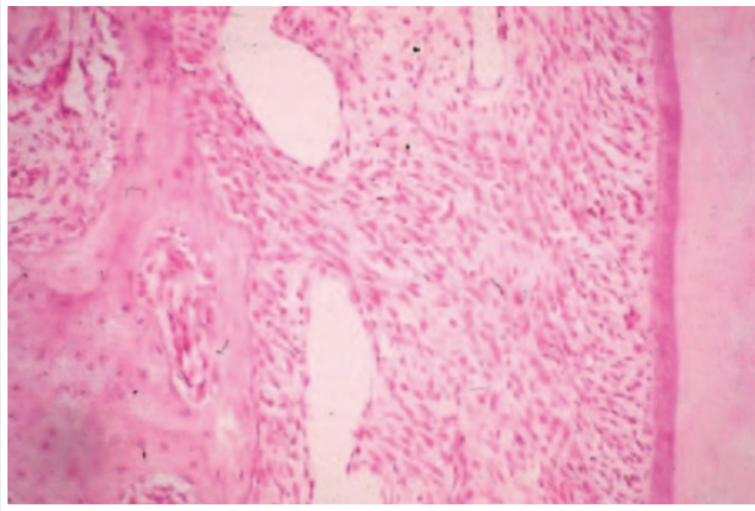

Fig. 2 This is a tension site where the bone adjacent to the periodontal ligament has surface lining osteoblasts and no sign of any osteoclasts. New bone is laid down as the tooth moves

\section{Intermittent forces appear to move teeth and stimulate bone remodelling more efficiently than continuous forces}

increase in ionised blood calcium within 1 hour. These finding have done much to unravel the final links between bone formation and resorption.

One other role that osteoblasts have in bone resorption is removal of the non-mineralised osteoid layer. In response to bone resorbing hormones, the osteoblast secretes MMPs which are responsible for removal of osteoid. This exposes the mineral layer to osteoclasts for resorption. It has been suggested that the mineral is also chemotactic for osteoclast recruitment and function.

How mechanical forces stimulate bone remodelling remains a mystery but some key facts are known. First, intermittent forces stimulate more bone remodelling than continuous forces. It is likely that during orthodontic tooth movement intermittent forces are generated because of 'jiggling' effects as teeth come into occlusal contact. Second, the key regulatory cell in bone metabolism is the osteoblast. It is therefore relevant to examine what effects mechanical forces have on these cells. The application of a force to a cell membrane triggers off a number of responses inside the cell and this is usually mediated by second messengers. It is known that cyclic AMP, inositol phosphates and intracellular calcium are all elevated by mechanical forces. Indeed the entry of calcium to the cell may come from G-protein controlled ion channels or release of calcium from internal cellular stores. These messengers will evoke a nuclear response which will either result in production of factors responsible for osteoclast recruitment and activation, or bone forming growth factors. An indirect pathway of activation also exists whereby membrane enzymes (phospholipase A2) make substrate (arachidonic acid) available for the generation of prostaglandins and leukotrienes. These compounds have both been implicated in tooth movement.

The main theories of tooth movement are now summarised:

\section{BIOMECHANCIAL ORTHODONTIC TOOTH MOVEMENT}

This theory simply states that mechanically distorting a cell membrane activates PLA2 making arachidonic acid available for the action of cyclo and lipoxygenase enzymes. This produces prostaglandins which feed back onto the cell membrane binding to receptors which then stimulate second messengers and elicit a cell response. Ultimately, these responses will include bone being laid down in tension sites and bone being resorbed at pressure sites. It is not clear how tissues discriminate between tension and pressure. It is worth remembering that cells which are rounded up show catabolic changes whereas flattened cells (? under tension) have anabolic effects.

\section{BONE BENDING, PIEZOELECTRIC AND MAGNETIC FORCES}

There was considerable interest in piezoelectricity as a stimulus for bone remodelling during the 1960s. This arose because it was noted that distortion of crystalline structures generated small electrical charges, which potentially may have been responsible for signalling bone changes associated with mechanical forces. The interest therefore in 'electricity' and bone was considerable.

Magnets have been used to provide the force needed for orthodontic tooth movement. Classically an unerupted tooth has a magnet attached to it and a second magnet is placed on an orthodontic appliance with the poles orientated to provide an attractive force. It is unlikely that the magnetic forces alone have any actions on tissues. If magnetic fields are broken (as in pulsed electromagnetic fields) then there is some evidence that tissues will respond. It is worth making the following points about the effects of magnetic and electric fields on tooth movement:

- The periodontal ligament is unlikely to transfer forces to bone. If the periodontal ligament is disrupted, orthodontic tooth movement still occurs 
- Magnetic fields alone have little, if any, effect on tissues

- Pulsed magnetic fields (which induce electric fields) can increase the rate and amount of tooth movement

- When an orthodontic force is applied, the tooth is displaced many times more than the periodontal ligament width. Bone bending must therefore occur in order to account for the tooth movement over and above the width of the periodontal ligament

- Physically distorting dry bone produces piezoelectric forces which have been implicated in tooth movement. Piezoelectric forces are those charges which develop as a consequence of distorting any crystalline structure. The magnitude of the charges is very small and there is some doubt whether they are sufficient to induce cellular change.

- It must also be remembered that in hydrated tissues, streaming potential and nerve impulses produce larger electrical fields and thus it is unlikely that piezoelectric forces alone are responsible for tooth movement. ${ }^{2}$

A wider application of the phenomenon of mechanically induced bone remodelling is seen where sutures are stretched. In young orthodontic patients the midline palatal suture can be split using rapid maxillary expansion techniques. The resulting tension generates new bone which fills in between the distracted maxillary shelves. A similar technique is also used to lengthen limbs. This

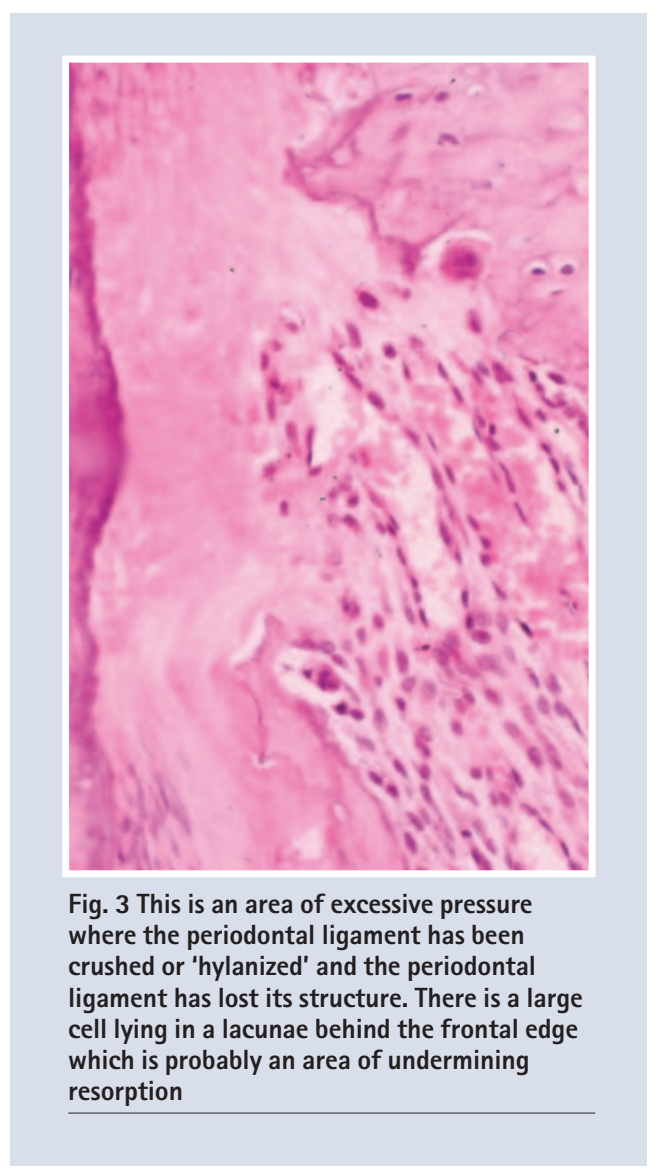

method, known as distraction osteogenesis, can be used in any situation where it is hoped that new bone will be generated. Originally this was described in Russia where many soldiers returning from war faced the problem of non-union limb fractures. Initially attempts were made to induce new bone formation by compressing bone ends. It was only when a patient inadvertently turned the screw for compression of bone ends in the wrong direction that it was noted excessive new bone formation was seen where bone ends were distracted rather than compressed.

This may also have application in patients whose sutures fuse prematurely (craniosynostoses such as Crouzon's or Aperts Syndrome). In this situation continued growth of the brain results in a characteristic appearance of the cranium but more importantly the eyes become protuberant with possible damage to the optic nerve. Treatment involves surgically opening the prematurely fused sutures and burring out to enable normal brain growth. If distraction forces are applied prior to this early fusion then bony infill could occur at a controlled rate. The phenomenon of pressure resulting in bone loss is also seen in pathological lesions. Much work was done to examine pressures within cystic lesions and to equate this with the rate of bone destruction. It is now recognised that cytokines and bone resorbing factors produced by cystic and malignant lesions are more likely to be responsible for the associated bone resorption.

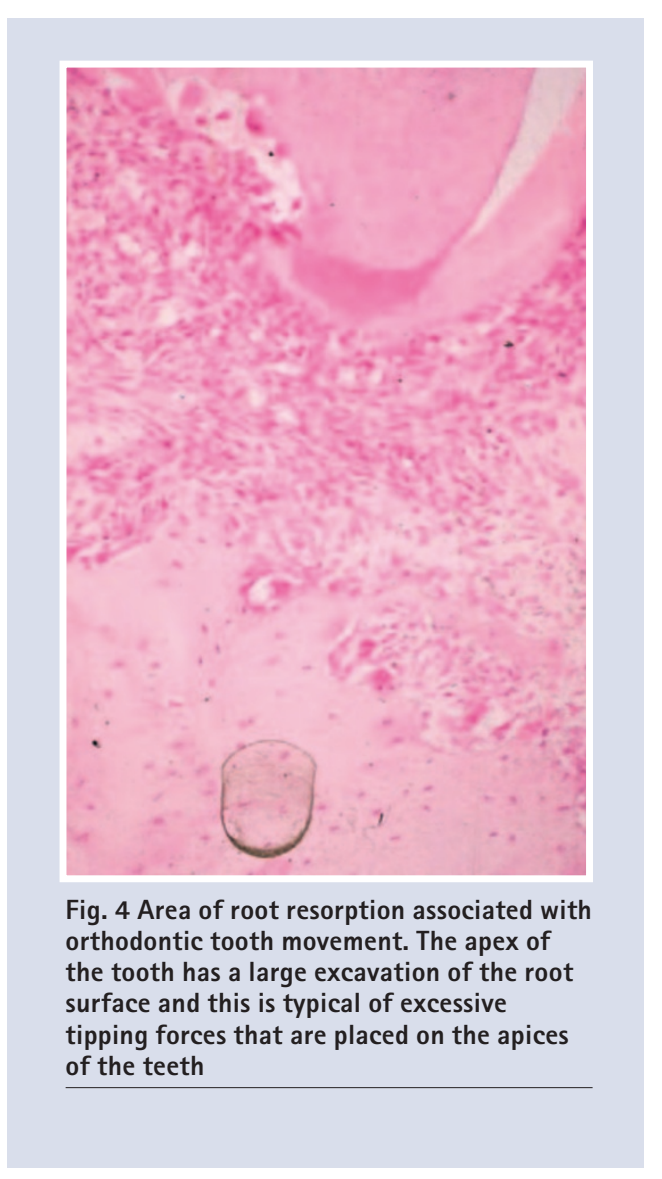

Tension results in bone formation, this can be used to generate new bone for digit lengthening or suture distraction 


\section{ROOT RESORPTION}

The ability to move teeth through bone is dependent on bone being resorbed and tooth roots remaining intact. It is highly probable that all teeth which have undergone orthodontic tooth movement exhibit some degree of microscopic root resorption (Fig. 4). Excessive root resorption is found in 3-5\% of orthodontic patients. Some teeth are more susceptible than others, upper lateral incisors can, on average, lose $2 \mathrm{~mm}$ of root length during a course of fixed orthodontic treatment. There are specific features of appliances which can increase the risk of root resorption. The following are considered risk factors:

- Fixed appliances

- Class II elastics

- Rectangular wires

- Orthognathic surgery

There is also some evidence that the use of functional appliances appears to cause less resorption than fixed appliances and may be used to reduce increased overjet where there are recognised risks of root resorption which include pre-existing features such as:

- Short roots

- Blunt root apices

- Thin conical roots

- Root filled teeth

- Teeth which have been previously traumatised

What prevents roots from resorbing is not known but the following have been suggested:
- Cementum has anti-angiogenic properties. This means blood vessels are inhibited from forming adjacent to cementum and osteoclasts have less access for resorption.

- Periodontal ligament fibres are inserted more densely in cementum than alveolar bone and thus osteoclasts have less access to the cemental layer.

- Cementum is harder than bone and more densely mineralised.

- Cemental repair may be by a material which is intermediate between bone and cementum. These semi-bone like cells may be more responsive to systemic factors such as parathyroid hormone and thus where roots are already short (and repaired with a bone/cementum like material) the teeth are more susceptible to further root resorption.

The exact reason why roots generally do not resorb is not known but without this property it would not be possible to move teeth orthodontically. A number of reviews are available which cover bone remodelling and tooth movement in greater depth. ${ }^{3,4}$

1. Waddington R J, Embery G, Samuels R H. Characterization of proteoglycan metabolites in human gingival fluid during orthodontic tooth movement. Arch Oral Biol 1994; 39: 361 368.

2. McDonald F. Electrical effects at the bone surface. Eur $J$ Orthod 1993; 15: 175-183.

3. Hill PA. Bone remodelling. Br J Orthod 1998; 25: 101-107.

4. Sandy J R, Farndale R W, Meikle M C. Recent advances in understanding mechanically-induced bone remodelling and their relevance to orthodontic theory and practice. $A m J$ Orthod Dento-fac Orthop 1993; 103: 212-222. 